Special Issue of the 6th International Congress \& Exhibition (APMAS2016), Maslak, Istanbul, Turkey, June 1-3, 2016

\title{
Degradation of Polyvinylpyrrolidone by Solution Plasma Process
}

\author{
A. AKYÜZ ${ }^{a, *}$ AND M. ÖZKAN ${ }^{b}$ \\ ${ }^{a}$ Mehmet Akif Ersoy University, Bucak EGTBMYO, Department of Electronics and Automation, \\ 15300 Burdur, Turkey \\ ${ }^{b}$ Afyon Kocatepe University, Department of Physics, 03200 Afyonkarahisar, Turkey
}

\begin{abstract}
The solution plasma technique, which has been recently invented by Takai and Saito, produces radicals, ions, UV photons, shock waves and metastable excited atoms. These generated active species are used as the source of some chemical reactions. The exact structure of the solution plasma is not well known at present, however it has been shown to be a versatile phenomenon for production of nanomaterials, sterilization and organic compound decomposition. Solution plasma technique has emerged as a great prospect for the chain scission (degradation) of polymers, to improve their solubility and physicochemical characteristics due to such advantages as absence of toxic materials, low temperature and usage of economical, simple equipment. In this study, polyvinylpyrrolidone, uncharged water soluble polymer of a known initial molecular weight, was continuously monitored using the continuous viscometry method, which gives the viscosity at any moment of the reaction, during the interaction of polyvinylpyrrolidone with plasma, for the first time in the literature.
\end{abstract}

DOI: 10.12693/APhysPolA.131.343

PACS/topics: 36.20.Fz, 52.77.-j, 66.20.-d

\section{Introduction}

Non-thermal or low temperature plasma in liquid, known as solution plasma process (SPP), continues to attract attention simply because of its application in numerous areas of work, such as nanoparticle synthesis, sterilization and decomposition reactions [1-5]. SPP has been also used for degradation of biopolymers. Wathanaphanit and Saito have described the effect of polymer concentration on the depolymerization of sodium alginate by SPP [6]. Tatiplapol et al. have studied the effect of SPP conditions, such as electrode type and pulse frequency, on degradation rate of chitosan [7]. Polyvinylpyrrolidone $(\mathrm{PVP}),\left(\mathrm{C}_{6} \mathrm{H}_{9} \mathrm{NO}\right)_{n}$, is a nonionic water-soluble polymer, which has drawn considerable attention over the last 20 years and has an extensive usages in pharmaceutical, medical and other industries. The usage of PVP in drug industry is mainly caused by its potential to produce complexes with various drugs, which makes them dispersible [8].

In the course of polymerization, it is surely not all the time possible to get a desired molecular weight of polymers. It is necessary to develop an effective and quick technique for getting the desired characteristics of polymers. A promising approach to obtain desired molecular weight of polymers will likely be based on polymeric chain scission, which is possible by chemical, biological and mechanical methods [9]. To the best of our knowledge, degradation of synthetic polymers by SPP has not yet been reported. In the present study, degradation

*corresponding author; e-mail: aakyuz@mehmetakif.edu.tr of PVP in aqueous solution by SPP was performed and continuously monitored by viscometry.

\section{Experimental procedure}

Polyvinylpyrrolidone (Sigma-Aldrich), having average molecular weight $1300000 \mathrm{~g} / \mathrm{mol}$ was used as-received. Water was distilled and filtered using Agilent $0.45 \mu \mathrm{m}$ econofilter. The plasma medium contained $2 \%$ methanol as a radical trap to suppresses the recombination of macro-radicals, formed by degradation. PVP concentration was $0.0005 \mathrm{~g} / \mathrm{g}$ in all experiments. Solutions were saturated by nitrogen bubbling prior to the plasma treatment.

The SPP system reported by Watthanaphanit was modified and used for degradation experiments [6]. A $50 \mathrm{ml}$ glass was used as the plasma vessel. Two tungsten needle electrodes (diameter of $1 \mathrm{~mm}$ ), covered by ceramic tubes, were fixed by the conical Teflon plugging. The distance gap between two electrodes was set to $1 \mathrm{~mm}$. The bipolar-DC power supply (Pekuris MPP HV-04) was used for discharge production. The pulsed electric discharge was generated at fixed voltage $(1.5 \mathrm{kV})$, pulse width $(2.5 \mu \mathrm{s})$ and frequency $(15 \mathrm{kHz})$. To minimize the increase of temperature, cold water was circulated through the vessel during plasma treatment.

Optical emission spectra (Ocean Optics USB 2000) was used to analyze the active species in the wave length range of 200-1100 nm. Optical fiber of the spectrometer was fixed at a distance of $1 \mathrm{~mm}$ right in front of the plasma vessel. Online viscometric monitoring technique has been described previously [10]. In the experiments, a peristaltic pump (Watson Marlow Sci 400) with silicone tubing was used to withdraw the PVP solution 
$(0.5 \mathrm{ml} / \mathrm{min})$ and then to pump the solution into the viscometer without dilution and to return it into the plasma vessel.

The volume in the plasma vessel was kept constant in order to avoid variation of degradation medium. Pressure variation $P$ is proportional to total solution viscosity $\eta$, based on Poisseuille's equation [10]. Preceding calibration was not need in the experiments, due to starting with the known molecular weight of PVP. Specific viscosities $\eta_{s p, t}$ at time $t$ (every $5 \mathrm{~s}$ ) are obtained from Eq. (1). In this equation $V_{t}$ represents the voltage signal and $P_{t}$ represents the pressure drop at time $t . V_{\mathrm{s}}, P_{\mathrm{s}}$ refer to solvent values. The Solomon-Ciuta formula (Eq. (2)) gives the intrinsic viscosity $\left(\eta_{\text {int }}\right)$ to a good approximation for one point measurements and is often used with pressure imbalance viscometers. Here $\eta_{\text {rel }}$ is the relative viscosity and $c$ is the polymer concentration. The plasma treatment is started with known molecular weight, the intrinsic viscosity data is used to determine the molecular weight during SPP using the Flory approximation (Eq. (3)) for good solvents. In Eq. (3), $M_{t}$ is molecular weight of PVP at treatment time $t, M_{0}$ is the initial molecular weight.

$$
\begin{aligned}
& \eta_{s p, t}=\left[\frac{V_{t}-V_{\mathrm{s}}}{V_{\mathrm{s}}}\right]=\left[\frac{P_{t}-P_{\mathrm{s}}}{P_{\mathrm{s}}}\right]=\left[\frac{\eta_{t}-\eta_{\mathrm{s}}}{\eta_{\mathrm{s}}}\right] . \\
& \eta_{\mathrm{int}}=\left[\sqrt{2\left(\eta_{s p}-\ln \left(\eta_{\mathrm{rel}}\right)\right.}\right] / c . \\
& \frac{M_{t}}{M_{0}}=\left(\frac{\eta_{\mathrm{int}, t}}{\eta_{\mathrm{int}, 0}}\right)^{1.25} .
\end{aligned}
$$

\section{Results and discussion}

Figure 1 shows the raw viscosity data for an experiment of plasma treatment of PVP. The viscosity of the solvent (water), of the PVP solution during the absence of discharge and during the SPP period are marked in

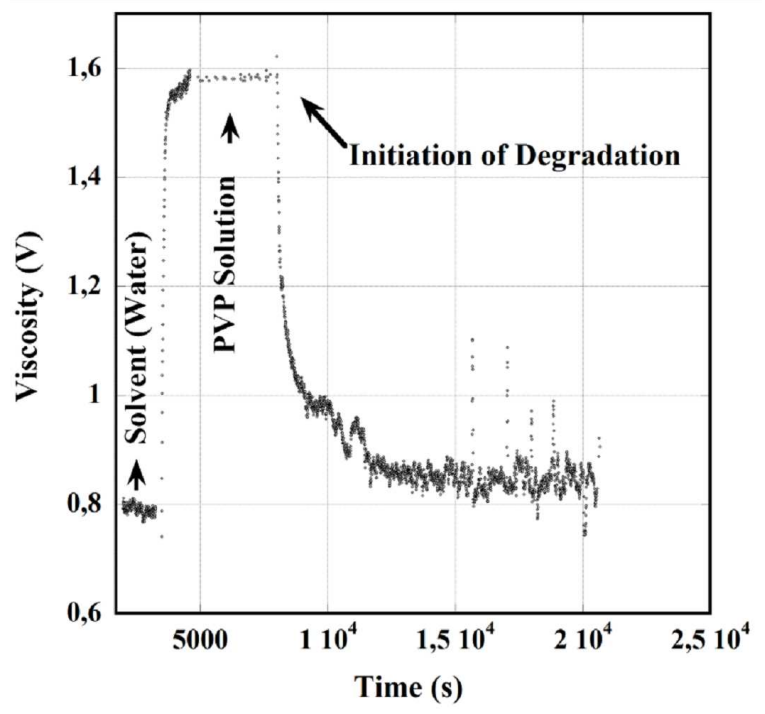

Fig. 1. Raw viscosity data. the figure. For about one hour water was pumped to the viscosimeter, before the PVP solution was introduced. Plasma treatment was started at about two hours after a stable voltage signal was obtained from the PVP solutions. The increase of the signal was due to introduction of the PVP and the decrease of signal, corresponding to initiation of discharge can be clearly seen in Fig. 1. To obtain details of the radicals during SPP, emission spectrum of treated (for $20 \mathrm{~min}$ ) sample is shown in Fig. 2. The radicals during plasma treatment were hydrogen radicals $(655,485$ and $430 \mathrm{~nm})$, oxygen radicals $(770,845 \mathrm{~nm})$ and $\mathrm{OH}$ radicals $(305 \mathrm{~nm})$. Figure 3a shows the time evolution of specific viscosity during SPP. It is clearly seen that the specific viscosity decreases initially, but finally reaches a limit value. The evolution of molecular weight (Fig. 3b) could be described by $\left(1 / M_{t}\right)-\left(1 / M_{0}\right)=k t$. Here $k$ represents the degradation constant equal to $6.32 \times 10^{-10} \mathrm{~s}^{-1}$. Figure shows that linear relationship exist between the $\left(1 / M_{t}\right)-\left(1 / M_{i n}\right)$ and treatment time. This can be explained by random chain scission of PVP occurring during the SPP.

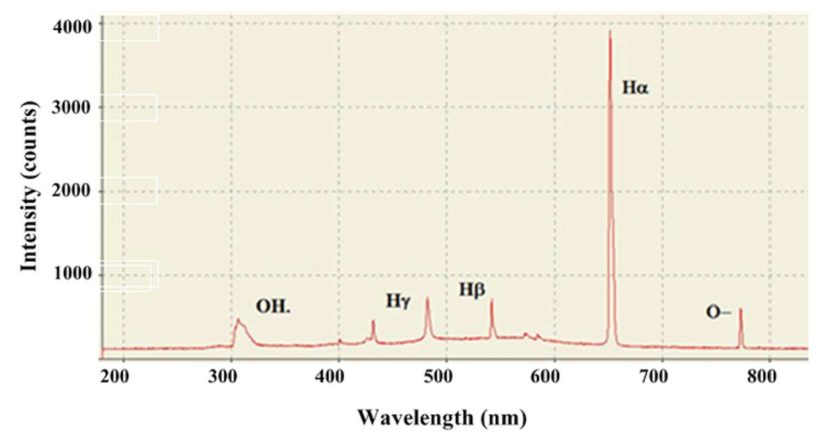

Fig. 2. Optical emission spectra of plasma-treated PVP solution (treatment time $20 \mathrm{~min}$ ).
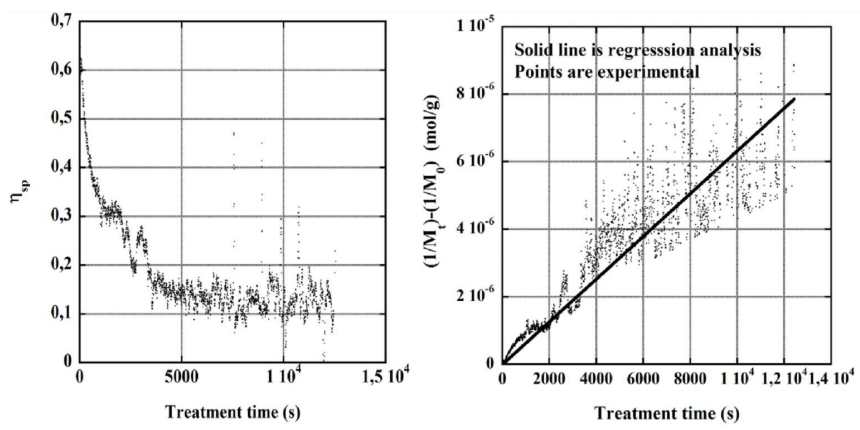

Fig. 3. (a) Specific viscosity evolution during SPP, (b) variation of inverse molecular weight with treatment time.

\section{Conclusions}

In the present study, the degradation of PVP in aqueous solution by SPP was studied at room temperature and under atmospheric pressure. It was observed that the viscosity and molecular weight of PVP solution has 
decreased significantly because of the plasma treatment. The study has also revealed that the degradation of PVP had continued to a limiting value. The use of the online monitoring technique for the first time has enabled viscosity data to be monitored continuously during the degradation of PVP by SPP. SPP can represent an effective method for the degradation of polymers in polymer industry.

\section{Acknowledgments}

This work was supported by the research grant of The Scientific and Technological Research Council of Turkey (TÜBİTAK) under the Project number 115Z421.

\section{References}

[1] G. Panomsuwan, N. Saito, T. Ishizaki, Electrochem. Commun. 59, 81 (2015).

[2] K. Hyun, T. Ueno, G. Panomsuwan, O.L. Li, Phys. Chem. Chem. Phys. 18, 10856 (2016).
[3] H. Lee, T. Ueno, N. Saito, J. Min. Met. Mat. S. 67, 2550 (2015).

[4] L. Cai, Y.Y. Wu, Y.H. Wu, S. Yamauti, N. Saito, Water Sci. Technol. 68, 923 (2013).

[5] N. Andreeva, T. Ishizaki, P. Baroch, N. Saito, Jpn J. Appl. Phys. 51, 126201 (2013).

[6] A. Watthanaphanit, N. Saito, Polym. Degrad. Stabil. 98, 1072 (2013).

[7] T. Tantiplapol, Y. Singsawat, N. Narongsil, S. Damrongsakkul, N. Saito, I. Prasertsung, Innov. Food Sci. Emerg. Technol. 32, 116 (2015).

[8] F. Haaf, A.Sanner, F. Straub, Polym. J. 17, 143 (1985).

[9] A.L. Prajapat, P.R. Gogate, Ultrason. Sonochem. 31, 371 (2016).

[10] A. Akyüz, O. Kamer, A. Giz, J. Macromol. Sci. A 50, 535 (2013) 